\title{
An Intracellular Delay-Differential Equation Model of the HIV Infection and Immune Control
}

\author{
T. Dumrongpokaphan ${ }^{a}$, Y. Lenbury ${ }^{b}$, R. Ouncharoen ${ }^{a, 1}$ and Y. Xu ${ }^{c}$ \\ ${ }^{a}$ Department of Mathematics, Faculty of Science, Chiangmai University \\ Chiangmai, 50200 Thailand \\ ${ }^{b}$ Department of Mathematics, Faculty of Science, Mahidol University \\ Bangkok, 10400 Thailand \\ ${ }^{c}$ Department of Mathematics, Syracuse University, Syracuse, NY 12344, USA
}

\begin{abstract}
Previous work has shown that intracellular delay needs to be taken into account to accurately determine the half-life of free virus from drug perturbation experiments [1]. The delay also effects the estimated value for the infected T-cell loss rate when we assume that the drug is not completely effective [19]. Models of virus infection that include intracellular delay are more accurate representations of the biological data.

We analyze a non-linear model of the human immunodeficiency virus (HIV) infection that considers the interaction between a replicating virus, CD4+ T-cell and the cytotoxic-lymphocytes (CTL). We then investigate the intracellular delay effect on the stability of the endemically infected steady state. Criteria are given to ensure that the infected steady state is asymptotically stable for all delays. Model analysis also allows the prediction of a critical delay $\tau_{c}$ below which the effector CTL can play a significant role in the immune control mechanism even when the basic reproduction number is high.
\end{abstract}

Key words: delay system, CD4+T-cell, the HIV infection and immune control AMS subject classification: 34A34, 37N25, 92B05

\footnotetext{
${ }^{1}$ Corresponding author. E-mail: rujira@chiangmai.ac.th.
} 


\section{Introduction}

In the last decade, mathematical models have proven to be valuable in understanding the dynamics of the HIV infection. Many simple mathematical approaches have been developed to explore the relation between antiviral immune responses, virus load and virus diversity infections with HIV. Moreover, mathematical models based on a firm understanding of biological interactions, can provide non-intuitive insights into the dynamics of host response to infectious agents.

A simple model for the interaction between the human immune system and HIV was developed by Perelson [24]. Later, Perelson et al. [23] extended this model and studied mathematically the model's behavior. It was observed there that the model exhibits many of the symptoms of AIDS seen clinically: the long latency period, low levels of free virus in the body, and the depletion of CD4+ T-cells. The model was constructed in the paper by considering four compartments: uninfected cells, latently infected cells, actively infected cells and free virus. The dynamics of these populations was also described in terms of a system of four ordinary differential equations.

The discrete and continuous time delays have been incorporated into biological models as proposed in many previous works $[11,14,18,26]$. It is well known that, delay-differential equations exhibit much more complicated dynamics than ordinary differential equations since a delay may cause a stable steady state to lose its stability and cause the population levels to oscillate. It was proposed by Perelson et al. [26] that there are two types of delay: a pharmacological delay and an intracellular delay. A pharmacological delay occurs between the ingestion of drug and its appearance within cells, and an intracellular delay occurs between the infection of a host cell and the emission of viral particles. Herz et al. [14] showed that the incorporation of a discrete intracellular delay in a HIV model would substantially shorten the estimate for the half-life of free virus. Mittler et al. [18] examined a related model and showed that a Gamma-distribution delay would be more accurate, rather than being discrete. They obtained better estimates for the viral clearance rate constant by assuming the drug to be completely effective. In a work of Grossman et al. [11], a model was proposed by introducing a delay in the cell death process, under the assumption that a productively infected cell died by the first order process. It also incorporated the feature that production of virus was delayed from the time of initial infection. Later Culshaw and Ruan [7] simplified the model proposed in the work of Perelson et al. [23] by considering only three components: the uninfected CD4+ T-cells, infected CD4+ T-cells, and free virus. The reduced model is thus more mathematically tractable, allowing theoretical analysis using the delay as the bifurcation parameter. A discrete delay was incorporated to the model to describe the time between infection of a CD4+ T-cell and the release of new viruses as proposed by Herz et al. [14]. It was shown by Nelson et al. [21], using a model with a discrete infection delay and constant target cell density, that if the assumption of completely effective drug was relaxed, then the estimated values of both the viral clearance rate and the rate of loss of productively infected $T$ cells were affected by the delay when the model was fitted to experimental data.

In this paper, we provide a detailed analytical study of a mathematical model of the interaction between infective virus, CD4+ T-cells, and CTL, which incorporates a discrete intracellular delay in time between infection of a CD4+ T-cell and the emission of viral particles on a cellular level as proposed in, say, Herz et al. [14], Tam [30], Nelson et al. [19], and Culshaw and Ruan [7]. 
Specifically, we consider the existence and stability of the infected steady state of the system.

We organize this paper in six sections. In section 2, we describe a background of the HIV infection and immune control mechanism. We study in section 3 the ordinary differential equation model for this problem, and in section 4 the model with delay. We present a numerical experiment in section 5. Finally, in section 6 , we make a conclusion based on our theoritical study and numerical results.

\section{Background}

In this section, we discuss the biological background of the problem to be studied. The material presented here is taken from [5], [6], [8], [14], [16], [18], [22], [23], [26], [27], and [32].

It is a well-known fact that viruses are intracellular parasites that depend on the host cell to survive and duplicate. The host cell can be damaged by the virus or by antibodies, cytokines, natural killer cells, and T-cells which are essential components of a normal immune response to the virus. The effective antiviral immune response depends on the amount of virus present, the infected tissues and the chronicity of the infection [32]. The virus load is an important factor of the release of new viruses. For example, in HIV-1 infections, virus load is correlated with pathogenicity, disease stage, and progression of disease [6, 27]. However, in most virus infections, cytotoxic T-lymphocytes play a critical role in antiviral defense by attacking infected cells and they are the main host immune factor that limits the extent of virus replication in vivo and thus determines the virus load [22].

The CD4+ T-cells, the most abundant white blood cells of the immune system (referred to as helper T-cells) are the main host of HIV. It has been discovered that HIV, although attacking many different cells, causes the most widespread damage on the CD4+ T-cells by causing their destruction and decline, and decreasing the ability of the immune system.

In HIV-1 infection, after potent antiretroviral therapy is initiated in patients, the level of free virus in distinct phases declines. Before initiation of therapy, for patients whose viral load is stationary, there is a period that lasts anywhere from 6 hours to a few days after initiation of therapy where little change is noticed in the virus concentration [26]. In this period the viral decay curve is said to have a 'shoulder'. The delay or shoulder is believed to be due to a number of processes. First, a pharmacological delay, a delay associated with the transport and intracellular processing of drug. Second, infected cells continue to produce virus if protease inhibitors are given but the virus is non-infectious. Thus, the virus concentration does not fall until there is a decrease in the number of virus producing cells. Third, if a reverse transcriptase inhibitor is given, cells that have already been infected by the virus and its RNA reverse transcribed, will continue to go through the set of intracellular events that slowly lead to virus production. After the shoulder, at the beginning the viral decline is very rapid and then one to two weeks after therapy initiation the rate of decline decreases [10, 14, 18, 20, 26].

Generally, the CD4 count (normally 1,000 per $\mu l$, with a range of $600-1,400$ per $\mu l$ [25]) is a good indicator for CD4 densities in blood. After HIV enters the body, it targets all the cells with CD4+ receptors, including the CD4+ T-cells then the gp 120 protein on the viral particle binds 
to the $\mathrm{CD} 4+$ receptors on the $\mathrm{CD} 4+\mathrm{T}$-cell and injects its core. After an intracellular delay the infected cell releases new viruses that can infect other CD4+ T-cells. The CD4 count falls during primary infection, then returns to near or lower than normal levels. Eventually, it slowly decreases, taking many years to reach the level of 200 per $\mu l$ that characterizes AIDS [3, 25].

\section{Ordinary Differential Equation Model}

Recently, in a work of Verotta and Schaedeli [31], simple models were developed taking into account the essential non-linearity of HIV dynamics. These models can be identified from routinely collected HIV-1 clinical data. The models are expressed as a system of differential equations as done when analyzing phamacokinetics/pharmacodynamic data, and the parameters of the models given viral load data were estimated by standard methods. Some of the estimated values of these parameters may be found in [2] and [5]. For example, the average number of virions produced per productively infected cell is 500 virions/cell, while the clearance rate of free virus is estimated at 0.95/day-cell [5]. The models also incorporate different factors associated with resurgence: the intrinsic non-linear HIV-1 dynamics, drug exposure and in particular compliance to treatment, and insurgence of resistant HIV-1 strains. However these models do not incorporate time delay.

We first describe a simple HIV model proposed in [31] physiologically by using the fact that there is no difference between infected and non-infected CD4+ T-cells when CD4+ T-cell is routinely counted in patients (see, for example, [1, 3, 15, 22, 31] for similar alternative models). The dynamic model includes the interaction of CD4+ T-cells, infective HIV, and cytotoxic-Tlymphocytes, CTLs. We use $T, V$, and $C$ to denote the population densities of CD4+ T-cells, infective HIV, and CTLs, respectively. Then $\frac{d T}{d t}$, $\frac{d V}{d t}$, and $\frac{d C}{d t}$ denote the rates of change in population densities of CD4+ T-cells, infective HIV, and CTLs, respectively, at time $t$. We are led to the following system model proposed by Verotta and Schaedeli [31]

$$
\begin{aligned}
& \frac{d T}{d t}=\delta_{1}-\delta_{2} V T-\delta_{3} T, \\
& \frac{d V}{d t}=\delta_{4} V T-\delta_{5} V-\delta_{6} V C, \\
& \frac{d C}{d t}=\delta_{7} V-\delta_{8} C,
\end{aligned}
$$

where the parameters are: the production rate of CD4+ T-cells $\left(\delta_{1}\right)$, the death rate of CD4+ T-cells due to the infection $\left(\delta_{2}\right)$, the natural death rate of CD4+ T-cells $\left(\delta_{3}\right)$, the production rate of virus $\left(\delta_{4}\right)$, and the death rate of virus $\left(\delta_{5}\right)$.

We note that the rate constant $\delta_{2}$ accounts for the death of CD4+T-cells due the direct virus infection as well as death due to CTLs that become effector (killer) cells in response to the target cells $\mathrm{T}$ becoming infected by the virus $\mathrm{V}$. Thus, this removal rate varies as the product of $\mathrm{V}$ and T. Through the destruction of infected cells before they can release a fresh crop of viruses, the infective virus is then reduced at the rate $\delta_{6} \mathrm{VC}$. The parameter $\delta_{7}$ is the rate of stimulation of CTLs by infective virus, and finally $\delta_{8}$ represents the natural death rate of CTLs. 
We now introduce new parameters,

$$
a_{1}:=\delta_{3}, \quad a_{2}:=\delta_{5}, \quad a_{3}:=\frac{\delta_{1} \delta_{4}}{\delta_{3} \delta_{5}}, \quad a_{4}:=\frac{\delta_{3} \delta_{6} \delta_{7}}{\delta_{2} \delta_{8}}, \quad a_{5}:=\delta_{8}
$$

where the parameter $a_{3}$ represents the basic reproductive ratio for the virus and $a_{4}$ represents the death rate of virus due to the immune response. By using these new parameters and introducing new variables

$$
x:=\frac{\delta_{3}}{\delta_{1}} T, \quad y:=\frac{\delta_{2}}{\delta_{3}} V, z:=\frac{\delta_{2} \delta_{8}}{\delta_{3} \delta_{7}} C,
$$

the differential equations above become

$$
\begin{aligned}
& \frac{d x}{d t}=a_{1}(1-x y-x), \\
& \frac{d y}{d t}=a_{2}\left(a_{3} x y-y\right)-a_{4} y z, \\
& \frac{d z}{d t}=a_{5}(y-z),
\end{aligned}
$$

By defining three nonlinear functions

$$
f(x, y, z):=a_{1}(1-x y-x), \quad g(x, y, z):=a_{2}\left(a_{3} x y-y\right)-a_{4} y z, h(x, y, z):=a_{5}(y-z),
$$

equations (3.1) become

$$
\frac{d x}{d t}=f(x, y, z), \quad \frac{d y}{d t}=g(x, y, z), \quad \frac{d z}{d t}=h(x, y, z) .
$$

A point $(\bar{x}, \bar{y}, \bar{z})$ is called a steady state of the system (3.2) if it is a constant solution of the equations

$$
f(\bar{x}, \bar{y}, \bar{z})=0, \quad g(\bar{x}, \bar{y}, \bar{z})=0, \quad h(\bar{x}, \bar{y}, \bar{z})=0 .
$$

The proof of the next proposition on the steady states of the system (3.2) is elementary so that it will be omitted.

Proposition 1. Suppose $a_{2}, a_{3}, a_{4}>0$

(i) If $a_{3} \leq 1$, then the nonnegative steady state of the system (3.2) is

$$
\left(\bar{x}_{0}, \bar{y}_{0}, \bar{z}_{0}\right)=(1,0,0) .
$$

(ii) If $a_{3}>1$, then the nonnegative steady states of the system (3.2) are that given in (3.4) and

$$
(\bar{x}, \bar{y}, \bar{z})=\left(\frac{1}{z^{*}+1}, z^{*}, z^{*}\right)
$$

where

$$
z^{*}=-\frac{1}{2}\left(1+\frac{a_{2}}{a_{4}}\right)+\frac{1}{2} \sqrt{\left(1+\frac{a_{2}}{a_{4}}\right)^{2}+4 \frac{a_{2}\left(a_{3}-1\right)}{a_{4}}} .
$$


Now, we consider a region close to the steady state and let

$$
x=\bar{x}+X, \quad y=\bar{y}+Y, \quad z=\bar{z}+Z .
$$

Expanding $f, g$, and $h$ in Taylor series expansions about $(\bar{x}, \bar{y}, \bar{z})$ then retaining only the linear terms we obtain the following linear system

$$
\left[\begin{array}{c}
\frac{d X}{d t} \\
\frac{d Y}{d t} \\
\frac{d Z}{d t}
\end{array}\right]=A\left[\begin{array}{c}
X \\
Y \\
Z
\end{array}\right]
$$

where $A$ denotes the Jacobian matrix of the model system evaluated at $(\bar{x}, \bar{y}, \bar{z})$, that is,

$$
A:=\left[\begin{array}{ccc}
-a_{1}(\bar{y}+1) & -a_{1} \bar{x} & 0 \\
a_{2} a_{3} \bar{y} & a_{2}\left(a_{3} \bar{x}-1\right)-a_{4} \bar{z} & -a_{4} \bar{y} \\
0 & a_{5} & -a_{5}
\end{array}\right] .
$$

We will study the stability of our model in terms of the eigenvalues of the matrix $A$ (see Plaat [28] for theoretical background).

Based on the theory of differential equations [28], we know that the steady state of the system (3.7) where $\operatorname{det} A \neq 0$, is stable if no eigenvalue of $\mathrm{A}$ has positive real part, and is asymptotically stable if all eigenvalues have negative real part.

Proposition 2. (i) Suppose $a_{1}, a_{2}, a_{3}, a_{5}>0$. The steady state (3.4) of the system (3.7) is asymptotically stable if $a_{3}<1$, and unstable if $a_{3}>1$.

(ii) If $a_{1}, a_{2}, a_{4}, a_{5}>0$ and $a_{3}>1$, then the steady state (3.5) of the system (3.7) is asymptotically stable.

Proof. We first remark that the characteristic equation of matrix $A$ is given by

$$
U_{0}(\lambda)=\lambda^{3}+\left(b_{1}+d_{1}\right) \lambda^{2}+\left(b_{2}+d_{2}\right) \lambda+b_{3}+d_{3}=0
$$

where

$$
\begin{aligned}
b_{1} & =a_{1}(1+\bar{y})+a_{2}+a_{5}+a_{4} \bar{z} \\
b_{2} & =a_{1}(1+\bar{y})\left(a_{2}+a_{5}+a_{4} \bar{z}\right)+a_{5}\left(a_{2}+a_{4}(\bar{y}+\bar{z})\right), \\
b_{3} & =a_{1} a_{5}(1+\bar{y})\left(a_{2}+a_{4}(\bar{y}+\bar{z})\right) \\
d_{1} & =-a_{2} a_{3} \bar{x} \\
d_{2} & =-a_{2} a_{3}\left(a_{1}+a_{5}\right) \bar{x} \\
d_{3} & =-a_{1} a_{2} a_{3} a_{5} \bar{x} .
\end{aligned}
$$

(i) In this case, we substitute the steady state (3.4) into the equations (3.8) and find that the characteristic equation has the form

$$
\left(\lambda+a_{1}\right)\left(\lambda+a_{2}\left(1-a_{3}\right)\right)\left(\lambda+a_{5}\right)=0 .
$$


Therefore, the eigenvalues of matrix $A$ are $\lambda_{1}=-a_{1}<0, \lambda_{2}=-a_{5}<0$ and $\lambda_{3}=-a_{2}(1-$ $a_{3}$ ). If $a_{3}<1$, clearly $\lambda_{3}<0$, which means that the steady state (3.4) is asymptotically stable. If $a_{3}>1$ then $\lambda_{3}>0$, we conclude that the steady state (3.4) is unstable.

(ii) Since $a_{3}>1$ the steady state (3.5) exists and $z^{*}>0$. By the Routh-Hurwitz criterion [9], it follows that all roots of the characteristic equation (3.8) have negative real parts if and only if

$$
b_{1}+d_{1}>0, \quad b_{3}+d_{3}>0, \quad\left(b_{1}+d_{1}\right)\left(b_{2}+d_{2}\right)-\left(b_{3}+d_{3}\right)>0 .
$$

It remains to verify that the conditions in (3.10) are satisfied.

By substituting (3.5) into equations (3.9), one obtains

$$
\begin{gathered}
b_{1}+d_{1}=a_{2}+a_{5}+a_{1}\left(1+z^{*}\right)-\frac{a_{2} a_{3}}{1+z^{*}}+a_{4} z^{*} \\
b_{3}+d_{3}=a_{1} a_{5}\left(1+z^{*}\right)\left(a_{2}+2 a_{4} z^{*}\right)-\frac{a_{1} a_{2} a_{3} a_{5}}{1+z^{*}}, \\
\beta:=\left(b_{1}+d_{1}\right)\left(b_{2}+d_{2}\right)-\left(b_{3}+d_{3}\right) \\
=-a_{1} a_{5}\left(1+z^{*}\right)\left(a_{2}+2 a_{4} z^{*}\right)+\frac{a_{1} a_{2} a_{3} a_{5}}{1+z^{*}} \\
+\left[a_{2}+a_{5}+a_{1}\left(1+z^{*}\right)-\frac{a_{2} a_{3}}{1+z^{*}}+a_{4} z^{*}\right] \\
\times\left[a_{5}\left(a_{2}+2 a_{4} z^{*}\right)-\frac{a_{2} a_{3}\left(a_{1}+a 5\right)}{1+z^{*}}+a_{1}\left(a_{2}+a_{5}+a_{4} z^{*}\right)\left(1+z^{*}\right)\right] .
\end{gathered}
$$

From (3.6), we can write

$$
a_{2} a_{3}=a_{4}\left(z^{*}\right)^{2}+a_{4} z^{*}+a_{2} z^{*}+a_{2} .
$$

Substituting equation (3.12) into the equations in (3.11) and simplifying, we obtain

$$
b_{1}+d_{1}=a_{1}\left(1+z^{*}\right)+a_{5}, \quad b_{3}+d_{3}=z^{*} a_{1} a_{5}\left(a_{2}+a_{4}\left(1+2 z^{*}\right)\right),
$$

and

$$
\beta=a_{1} a_{5}^{2}\left(1+z^{*}\right)+a_{4} a_{5}^{2} z^{*}+a_{1}^{2}\left(1+z^{*}\right)\left(a_{2} z^{*}+a_{4}\left(z^{*}\right)^{2}+a_{5}\left(1+z^{*}\right)\right),
$$

from which we see that condition (3.10) is satisfied. Thus, the steady state (3.5) is asymptotically stable.

We now interpret the results of Proposition 2 in the biological sense. When $a_{3}<1$, the basic reproduction ratio for the virus is at a controlled level which means the virus is unsuccessful in escaping from the specific immune response, CTL. Part ( $i$ ) of Proposition 2 ensures that if $a_{3}<1$ then the virus is unable to maintain the infection, and will become extinct as time passes. The CD4+ T-cell population will converge to the ratio $\delta_{1} / \delta_{3}$.

Changes in the basic reproduction ratio $a_{3}$ can have an influential effect on virus load even if the virus is not close to extinction, because a strong CTL response results in a low virus load. If the virus load $(V)$ is sufficiently small, then the total death rate of CD4+ T-cell due to the 
infection $\left(\delta_{2} V T\right)$ is small compared to the total loss of CD4+ T-cell $\left(\delta_{3} T\right)$. Thus, the virus weakly affects the steady state density of CD4+ T-cell. By this model, patients with weak CTL responses should have a smaller reduction in steady state virus load than those with strong CTL response. According to the above reason, if the physical parameters $\left(\delta_{1}, \delta_{3}, \delta_{4}, \delta_{5}\right)$ can be maintained so that the condition $a_{3}<1$ in Proposition $2(i)$ is satisfied, for example through vaccination and post-exposure immunization, then it may be possible to prevent progression towards AIDS.

However, by the second statement of part (i) and part (ii) of Proposition 2, if $a_{3}>1$, that is, the basic reproduction ratio for the virus is not in a controlled range, then the virus can establish an infection and the uninfected steady state (3.4) loses its stability while the infected steady state (3.5) comes into existence and is stable. The long asymptomatic period between infection and collapse of the immune system is the duration required for the virus population to evolve into full blown AIDS.

\section{Delay Differential Equation Model}

The mathematical model presented in the previous section does not incorporate any time delay in HIV proliferation. Virus production may lag by an intracellular time delay $\tau$ between the infection of a cell and the emission of viral particles. In many aspects of viral dynamics such a delay may be of no significance. However, as shown by Nelson and Perelson [20], when the drug efficacy is less than perfect, the intracellular delay will be of crucial importance. It shall be seen below that incorporation of intracellular delay leads to insightful interpretation of the analytical results which has important implications on therapeutic options and drug development.

Nelson and Perelson [20] developed and analyzed a set of models that include intracellular delays, combination antiretroviral therapy, and the dynamics of both infected and uninfected T cells. It was shown that when the drug efficacy is less than perfect the estimated value of the loss rate of productively infected $\mathrm{T}$ cell is increased when the data is fitted with delay models compared to the values estimated with a non-delay model. Their work extended the ability of models to describe the relevant biological process and addressed the implications of ignoring the intracellular delays that are part of the viral life cycle. However, their model did not take into account the role of Cytotoxic T Lymphocytes that play an intricate part in the immunological mechanism as will be seen in our later discussion.

We therefore modify the model given by (3.2) proposed by Verotta and Schaedeli [31]. The new model assumes a delay, $\tau$, from the time of initial infection until the production of new virus particles. To describe the delay in mathematical terms, we define a translation operator as follows. For a real-valued function $s$ and for $\tau \geq 0$, we define the translation operator $T_{\tau}$ by

$$
\left(T_{\tau} s\right)(t)=s(t-\tau), \quad t \geq 0,
$$

and for notational convenience we let

$$
x_{1}:=T_{\tau} x, \quad y_{1}:=T_{\tau} y, \quad z_{1}:=T_{\tau} z .
$$

We then obtain the new system with delay given by 


$$
\begin{gathered}
\frac{d x}{d t}=a_{1}(1-x y-x), \\
\frac{d y}{d t}=a_{2}\left(a_{3} x_{1} y_{1}-y\right)-a_{4} y z \\
\frac{d z}{d t}=a_{5}(y-z) .
\end{gathered}
$$

The term $a_{2} a_{3} x_{1} y_{1}$ reflects a finite time lag between infection of a CD4+ T-cell and production of new virus particles in the rate equation for $y$, which describes the change of the dynamical variable $y$ at time $t$ that depends on the term evaluated at earlier time $t-\tau$.

System (4.1) is a special case of the general system

$$
\begin{aligned}
& \frac{d x}{d t}=F\left(x, y, z, x_{1}, y_{1}, z_{1}\right), \\
& \frac{d y}{d t}=G\left(x, y, z, x_{1}, y_{1}, z_{1}\right), \\
& \frac{d z}{d t}=H\left(x, y, z, x_{1}, y_{1}, z_{1}\right),
\end{aligned}
$$

for functions $F, G$ and $H$ dependent on $x, y, z$ and their delays, with the following identification.

$$
\begin{gathered}
F\left(x, y, z, x_{1}, y_{1}, z_{1}\right):=a_{1}(1-x y-x), \\
G\left(x, y, z, x_{1}, y_{1}, z_{1}\right):=a_{2}\left(a_{3} x_{1} y_{1}-y\right)-a_{4} y z, \\
H\left(x, y, z, x_{1}, y_{1}, z_{1}\right):=a_{5}(y-z) .
\end{gathered}
$$

Note that when a function $s$ is constant, we have the relation

$$
\frac{d s}{d t}=\frac{d s_{1}}{d t}
$$

where $s_{1}=T_{\tau} s$. Taking this into consideration, we define the steady state for a delay system. A point $(\bar{x}, \bar{y}, \bar{z})$ is called a steady state of the system (4.2) if it is a constant solution of the equations

$$
F(\bar{x}, \bar{y}, \bar{z}, \bar{x}, \bar{y}, \bar{z})=0, \quad G(\bar{x}, \bar{y}, \bar{z}, \bar{x}, \bar{y}, \bar{z})=0, \quad H(\bar{x}, \bar{y}, \bar{z}, \bar{x}, \bar{y}, \bar{z})=0 .
$$

In the next proposition we consider the steady states of the system (4.1).

Proposition 3. If $a_{2}, a_{3}, a_{4}>0$ then the steady state solutions of the system (4.1) is the same as the steady states of the system (3.2).

Proof. By equation (4.3), we conclude that

$$
F(\bar{x}, \bar{y}, \bar{z}, \bar{x}, \bar{y}, \bar{z})=f(\bar{x}, \bar{y}, \bar{z}), \quad G(\bar{x}, \bar{y}, \bar{z}, \bar{x}, \bar{y}, \bar{z})=g(\bar{x}, \bar{y}, \bar{z}), \quad H(\bar{x}, \bar{y}, \bar{z}, \bar{x}, \bar{y}, \bar{z})=h(\bar{x}, \bar{y}, \bar{z}) .
$$

This implies that systems (4.1) and (3.2) have the same steady states. 
Now, consider a neighborhood close to steady state solutions and let

$$
x=\bar{x}+X, y=\bar{y}+Y, \quad z=\bar{z}+Z, \quad x_{1}=\bar{x}+X_{1}, \quad y_{1}=\bar{y}+Y_{1}, \quad z_{1}=\bar{z}+Z_{1} .
$$

Expanding $F, G$ and $H$ in the Taylor series about the point $(\bar{x}, \bar{y}, \bar{z})$, and retaining only the linear terms, we arrive at

$$
\begin{gathered}
\frac{d X}{d t}=-a_{1}(\bar{y}+1) X-a_{1} \bar{x} Y, \\
\frac{d Y}{d t}=a_{2} a_{3} \bar{y} X_{1}+\left(a_{2}\left(a_{3} \bar{x} Y_{1}-Y\right)-a_{4} \bar{z} Y\right)-a_{4} \bar{y} Z, \\
\frac{d Z}{d t}=a_{5} Y-a_{5} Z .
\end{gathered}
$$

Consider solutions in the form

$$
\left[\begin{array}{l}
x(t) \\
y(t) \\
z(t)
\end{array}\right]=C_{0} e^{\lambda t}
$$

where $C_{0}$ is a constant vector [16]. In this case, we have

$$
\left[\begin{array}{l}
x_{1} \\
y_{1} \\
z_{1}
\end{array}\right]=e^{-\lambda \tau}\left[\begin{array}{l}
x \\
y \\
z
\end{array}\right]
$$

and the system (4.5) can be rewritten as the linear system

$$
\left[\begin{array}{c}
\frac{d X}{d t} \\
\frac{d Y}{d t} \\
\frac{d Z}{d t}
\end{array}\right]=\left[\begin{array}{ccc}
-a_{1}(\bar{y}+1) & -a_{1} \bar{x} & 0 \\
a_{2} a_{3} \bar{y} e^{-\lambda \tau} & a_{2}\left(a_{3} \bar{x} e^{-\lambda \tau}-1\right)-a_{4} \bar{z} & -a_{4} \bar{y} \\
0 & a_{5} & -a_{5}
\end{array}\right]\left[\begin{array}{c}
X \\
Y \\
Z
\end{array}\right]
$$

Let

$$
B:=\left[\begin{array}{ccc}
-a_{1}(\bar{y}+1) & -a_{1} \bar{x} & 0 \\
a_{2} a_{3} \bar{y} e^{-\lambda \tau} & a_{2}\left(a_{3} \bar{x} e^{-\lambda \tau}-1\right)-a_{4} \bar{z} & -a_{4} \bar{y} \\
0 & a_{5} & -a_{5}
\end{array}\right]
$$

be the coefficient matrix of the linear system with $\lambda$ being an eigenvalue of the matrix $B$. Next, we will investigate the stability of the delay system in terms of the eigenvalues of the matrix $B$. We recall the following definition [12].

Definition 1. Let $\left(x_{0}, y_{0}, z_{0}\right)$ be a steady state solution of

$$
\frac{d x}{d t}=F\left(x, y, z, x_{1}, y_{1}, z_{1}\right), \frac{d y}{d t}=G\left(x, y, z, x_{1}, y_{1}, z_{1}\right), \frac{d z}{d t}=H\left(x, y, z, x_{1}, y_{1}, z_{1}\right)
$$


We say that $\left(x_{0}, y_{0}, z_{0}\right)$ is stable if for every $\epsilon>0$ there is $a \delta>0$ such that, for every solution $(x, y, z)$, if

$$
\left[x\left(t_{0}\right)-x_{0}\right]^{2}+\left[y\left(t_{0}\right)-y_{0}\right]^{2}+\left[z\left(t_{0}\right)-z_{0}\right]^{2}<\delta^{2}
$$

for some $t_{0} \in\left[t_{1}-\tau, t_{1}\right]$, then

$$
\left[x(t)-x_{0}\right]^{2}+\left[y(t)-y_{0}\right]^{2}+\left[z(t)-z_{0}\right]^{2}<\epsilon^{2}
$$

for all $t>t_{1}$. If $\left(x_{0}, y_{0}, z_{0}\right)$ is not stable, we say it is unstable. If $\left(x_{0}, y_{0}, z_{0}\right)$ is stable, and if $\delta$ can be chosen so that (4.6) implies that $(x(t), y(t), z(t)) \rightarrow\left(x_{0}, y_{0}, z_{0}\right)$ as $t \rightarrow \infty$, we say that $\left(x_{0}, y_{0}, z_{0}\right)$ is asymptotically stable.

From [12], we remark that the steady state $(\bar{x}, \bar{y}, \bar{z})$ is stable if no eigenvalue of $B$ has positive real part, and is asymptotically stable if all eigenvalues have negative real parts. The following analysis follows in a similar fashion as in the work by Ruan and Wei [29].

Proposition 4. Suppose that $a_{1}, a_{2}, a_{3}, a_{5}>0$.

(i) If $a_{3}<1$, then the steady state (3.4) of the system (4.1) is asymptotically stable for $\tau \geq 0$.

(ii) If $a_{3}>1$, then the steady state (3.4) of the system (4.1) is unstable for $\tau \geq 0$.

Proof. We first remark that the characteristic equation of matrix $B$ is given by

$$
U(\lambda)=\lambda^{3}+b_{1} \lambda^{2}+b_{2} \lambda+b_{3}+\left(d_{1} \lambda^{2}+d_{2} \lambda+d_{3}\right) e^{-\tau \lambda}=0 .
$$

When $\tau=0$, that is, there is no delay, this proposition reduces to Proposition 2. It remains to consider the case when $\tau>0$.

Substituting the steady state (3.4) into the equation (4.7), we find that the characteristic equation has the form

$$
\left(\lambda+a_{1}\right)\left(\lambda+a_{2}-a_{2} a_{3} e^{-\lambda \tau}\right)\left(\lambda+a_{5}\right)=0 .
$$

At the steady state (3.4), matrix $B$ has three eigenvalues $\lambda_{1}=-a_{1}<0, \lambda_{2}=-a_{5}<0$, and $\lambda_{3}$ satisfying the equation

$$
\lambda_{3}+a_{2}\left(a_{3} e^{-\tau \lambda_{3}}-1\right)=0 .
$$

To find the location of the eigenvalue $\lambda_{3}$, we introduce a function

$$
u(t):=t+a_{2}-a_{2} a_{3} e^{-\tau t}, \quad t \in R .
$$

By differentiation, we obtain

$$
u^{\prime}(t)=1+a_{2} a_{3} \tau e^{-\tau t},
$$

which is always positive. We also observe that

$$
\lim _{t \rightarrow-\infty} u(t)=-\infty, \lim _{t \rightarrow \infty} u(t)=\infty .
$$

Hence, the function $u$ has a unique zero. Note that

$$
u(0)=a_{2}\left(1-a_{3}\right) .
$$

If $a_{3}<1$, we conclude that $u(0)>0$ and thus, $\lambda_{3}<0$. In this case, the steady state (3.4) is asymptotically stable for all $\tau>0$. If $a_{3}>1$, we conclude that $u(0)<0$ and thus, $\lambda_{3}>0$. This ensures that the steady state (3.4) is unstable for all $\tau>0$. 
Recall that for the system (3.2), the steady state (3.5) is stable for the parameter values satisfying conditions in Proposition 2 (ii). Here, we are interested in determining whether there exists a critical delay $\tau_{c}>0$ in terms of $b_{1}, b_{2}, b_{3}, d_{1}, d_{2}$ and $d_{3}$ so that $\operatorname{Re}(\lambda)>0$ for $\tau>\tau_{c}$. In other words, $\tau_{c}$ is the value of $\tau$ such that $\operatorname{Re}(\lambda)=0$, at which the transition from stability to instability occurs. We will determine the conditions on the parameters to ensure that the steady state (3.5) of the delay system (4.1) is still stable by considering equation (4.7) as a complex variable mapping problem.

For the steady state (3.5), if we let $\lambda(\tau)=\alpha(\tau)+i \omega(\tau)$, where $\alpha$ and $\omega$ are real, then we have $\alpha(0)<0$. By continuity of $\alpha, \alpha(\tau)<0$ for values of $\tau$ such that $0 \leq \tau<\tau_{c}$ for some $\tau_{c}>0$. Therefore, the steady state remains stable for these values of $\tau$. Now, equation (4.7) becomes

$$
\begin{aligned}
& b_{3}+b_{2} \alpha+b_{1} \alpha^{2}+\alpha^{3}-b_{1} \omega^{2}-3 \alpha \omega^{2}+i\left(b_{2} \omega+2 b_{1} \alpha \omega+3 \alpha^{2} \omega-\omega^{3}\right) \\
& +e^{-\tau \alpha}\left[\left(d_{2} \omega+2 d_{1} \alpha \omega\right) \sin \omega \tau+\left(d_{3}+d_{2} \alpha+d_{1} \alpha^{2}-d_{1} \omega^{2}\right) \cos \omega \tau\right] \\
& +i e^{-\tau \alpha}\left[\left(d_{1} \omega^{2}-d_{3}-d_{2} \alpha-d_{1} \alpha^{2}\right) \sin \omega \tau+\left(d_{2} \omega+2 d_{1} \alpha \omega\right) \cos \omega \tau\right]=0
\end{aligned}
$$

Suppose $\alpha\left(\tau_{c}\right)=0$ for some $\tau_{c}>0$, and $\alpha(\tau)<0$ for $0 \leq \tau<\tau_{c}$, then the steady state (3.5) may lose stability at $\tau=\tau_{c}$ or $\lambda=i \omega\left(\tau_{c}\right)$. In fact, $i \omega$ is a root of equation (4.7) if and only if

$$
-i \omega^{3}-b_{1} \omega^{2}+i b_{2} \omega+b_{3}+\left(-d_{1} \omega^{2}+i d_{2} \omega+d_{3}\right)(\cos \omega \tau-i \sin \omega \tau)=0 .
$$

Equating real parts and imaginary parts of the right side of (4.9) to zero, one obtains

$$
\begin{aligned}
& d_{2} \omega \sin \omega \tau+\left(d_{3}-d_{1} \omega^{2}\right) \cos \omega \tau=b_{1} \omega^{2}-b_{3} . \\
& d_{2} \omega \cos \omega \tau-\left(d_{3}-d_{1} \omega^{2}\right) \sin \omega \tau=\omega^{3}-b_{2} \omega
\end{aligned}
$$

Adding up the squares of (4.10) and (4.11), one obtains

$$
u(\omega):=\omega^{6}+\left(b_{1}^{2}-2 b_{2}-d_{1}^{2}\right) \omega^{4}+\left(b_{2}^{2}-2 b_{1} b_{3}+2 d_{1} d_{3}-d_{2}^{2}\right) \omega^{2}+b_{3}^{2}-d_{3}^{2}=0 .
$$

To simplify equation (4.12), we introduce the quantities

$$
m:=\omega^{2}, \quad p:=b_{1}^{2}-2 b_{2}-d_{1}^{2}, \quad q:=b_{2}^{2}-2 b_{1} b_{3}+2 d_{1} d_{3}-d_{2}^{2}, \quad r:=b_{3}^{2}-d_{3}^{2}
$$

Hence, equation (4.12) reduces to

$$
K(m)=m^{3}+p m^{2}+q m+r=0 .
$$

Lemma 1. Let $\tau>0$. Suppose that equation (4.13) has no positive roots. Then, all roots of equation (4.7) have negative real parts.

Proof. Since equation (4.13) has no positive roots, any real number $\omega$ is not a root of equation (4.12). This ensures that any real number $\omega$ is not a root of equation (4.9). Hence, for any real number $\omega$, the value $i \omega$ is not a root of equation (4.7), which implies that there is no $\tau_{c}$ such that

$$
\lambda\left(\tau_{c}\right)=i \omega\left(\tau_{c}\right)
$$

is a root of equation (4.7). Because of Proposition 2, for a root of (4.7) corresponding to $\tau=0$, $\operatorname{Re}(\lambda(0))<0$. Since $\operatorname{Re}(\lambda(\tau))$ is a continuous function of $\tau$, we conclude that all roots of (4.7) have negative real parts. 
We next present conditions that ensure equation (4.13) has a positive root or has no positive roots. To this end, we differentiate

$$
K^{\prime}(m)=3 m^{2}+2 p m+q
$$

and observe that equation

$$
3 m^{2}+2 p m+q=0
$$

has the roots

$$
M_{0}:=\frac{-p+\sqrt{p^{2}-3 q}}{3}, \quad M_{1}:=\frac{-p-\sqrt{p^{2}-3 q}}{3} .
$$

Lemma 2. (i) If either (a) $r<0$, or (b) $r \geq 0, p^{2}-3 q>0, p<0$, and $K\left(M_{0}\right)<0$, then equation (4.13) has a positive root.

(ii) If $r \geq 0$ and $p^{2}-3 q<0$, then equation (4.13) has no positive roots.

Proof. (i) Suppose that condition ( $a$ ) holds, that is, $r<0$. Then, we have that $K(0)=r<0$. On the other hand, since

$$
\lim _{m \rightarrow \infty} K(m)=\infty
$$

by the intermediate value theorem, equation (4.13) must have a positive root $t_{0}$, that is, $K\left(t_{0}\right)=0$.

Now, suppose that condition $(b)$ holds. Since $r \geq 0, p<0$, and $p^{2}-3 q<0$, we find that $M_{0}$ is real and $M_{0}>0$. Since $K(0)=r \geq 0$ and $K\left(M_{0}\right)<0$, again by the intermediate value theorem, $K$ has a zero between the origin and $M_{0}$.

(ii) Since $q>\frac{1}{3} p^{2}$, both zeros $M_{0}$ and $M_{1}$ of $K^{\prime}$ are not real. That is, $K^{\prime}(m)=0$ has no real root. Noting that

$$
K^{\prime}(0)=q>\frac{1}{3} p^{2} \geq 0
$$

we conclude that the quadratic polynomial $K^{\prime}$ is strictly positive on the real numbers. This implies that $K$ is increasing on the real numbers. Moreover, since $K(0)=r \geq 0$, we observe that $K(m)$ does not vanish for $m>0$ and thus, equation (4.13) has no positive roots.

In addition, Lemma 2(ii) implies that there is no $\omega$ such that $i \omega$ is a solution of the characteristic equation (4.7). Therefore, the real parts of all the eigenvalues of $B$ are negative for all delay $\tau \geq 0$

Summarizing the above analysis we can write down the following theorem.

Theorem 1. Suppose that $a_{3}>1, a_{1}, a_{2}, a_{4}, a_{5}>0, r \geq 0$ and $p^{2}-3 q<0$. Then, the infected steady state (3.5) of the delay system (4.1) is asymptotically stable for all $\tau \geq 0$.

Proof. Since $U(\lambda)=U_{0}(\lambda)$ for $\tau=0$, by part (ii) of Proposition 2, all real parts of eigenvalue of $B$ are negative. By part (ii) of Lemma 2, equation (4.13) has no positive roots. Lemma 1 ensures that all roots of (4.7) have negative real parts for $\tau>0$. Therefore, the infected steady state (3.5) of the delay system (4.1) is asymptotically stable for all $\tau \geq 0$.

In the following theorem, let us introduce the Hopf bifurcation theorem (cf., Hassard et al. [13]). 
Theorem 2. Consider the 3-dimensional autonomous system of differential equations given by

$$
\begin{aligned}
\frac{d x}{d t} & =\boldsymbol{F}(x, y, z, \tau), \\
\frac{d y}{d t} & =\boldsymbol{G}(x, y, z, \tau), \\
\frac{d z}{d t} & =\boldsymbol{H}(x, y, z, \tau) .
\end{aligned}
$$

which depends on the real parameter $\tau$. If

(i) $\boldsymbol{F}(\bar{x}, \bar{y}, \bar{z}, \tau)=\boldsymbol{G}(\bar{x}, \bar{y}, \bar{z}, \tau)=\boldsymbol{H}(\bar{x}, \bar{y}, \bar{z}, \tau)=0$ for $\tau$ in an open interval containing $\tau_{c}$, and $(\bar{x}, \bar{y}, \bar{z})$ is a steady state solution of (4.15),

(ii) $\boldsymbol{F}, \boldsymbol{G}, \boldsymbol{H}$ are analytic in $x, y, z$ and $\tau$ in a neighborhood of $\left(\bar{x}, \bar{y}, \bar{z}, \tau_{c}\right)$,

(iii) the Jacobian matrix of (4.15) at $(\bar{x}, \bar{y}, \bar{z}, \tau)$ has a pair of complex conjugate eigenvalues $\lambda$ and $\bar{\lambda}$ such that

$$
\lambda(\tau)=\alpha(\tau)+i \omega(\tau)
$$

where

$$
\omega\left(\tau_{c}\right)=\omega_{c}>0, \alpha\left(\tau_{c}\right)=0,\left.\frac{d \alpha(\tau)}{d \tau}\right|_{\tau=\tau_{c}} \neq 0,
$$

(iv) the remaining eigenvalue of the Jacobian matrix at $\left(\bar{x}, \bar{y}, \bar{z}, \tau_{c}\right)$ has strictly negative real part, then the system (4.15) has a family of periodic solutions: there is an $\epsilon_{H}>0$ and an analytic function $\tau^{H}(\epsilon)=\Sigma_{2}^{\infty} \tau_{i}^{H} \epsilon^{i}\left(0<\epsilon<\epsilon_{H}\right)$ such that for each $\epsilon \in\left(0, \epsilon_{H}\right)$ there exists a periodic solution $p_{\epsilon}(t)$ occurring for $\tau=\tau^{H}(\epsilon)$. The period $T^{H}(\epsilon)$ of $p_{\epsilon}(t)$ is an analytic function

$$
T^{H}(\epsilon)=\frac{2 \pi}{\omega_{c}}\left(1+\Sigma_{2}^{\infty} T_{i}^{H} \epsilon^{i}\right)\left(0<\epsilon<\epsilon_{H}\right) .
$$

Next, we will provide the conditions on the parameters to ensure that the Hopf bifurcation occurs. Suppose conditions in Lemma 2(ia) hold, then equation (4.13) has a positive root. We denote, without loss of generality the positive roots of (4.13) by $m_{j}, j \in\{0\}$ or $j \in\{0,1,2\}$ depending on the number of positive roots (4.13)has. Equation(4.12), therefore, has at most six roots, $\pm \sqrt{m_{j}}$ for $j=0,1,2$.

If the solution of the equation (4.9) exists, it is among these $\pm \sqrt{m_{j}}$ for $j=0,1,2$. If $\lambda=i \omega$ is a root of equation (4.7) so is $-i \omega$.

Substituting $\omega_{j}$ into equations (4.10) and (4.11) and solving for $\tau$, we obtain

$$
\tau_{j}^{(n)}=\frac{1}{\omega_{j}} \arcsin \left[\frac{d_{1} \omega_{j}^{5}+\left(b_{1} d_{2}-d_{3}-d_{1} b_{2}\right) \omega_{j}^{3}+\left(d_{3} b_{2}-b_{3} d_{2}\right) \omega_{j}}{d_{2}^{2} \omega_{j}^{2}+\left(d_{3}-d_{1} \omega_{j}^{2}\right)^{2}}\right]+\frac{2 \pi(n-1)}{\omega_{j}}
$$

where $j=0,1,2$ and $n=1,2, \ldots$ 
Now, let $\tau_{c}>0$ be the smallest of such $\tau$ for which $\alpha\left(\tau_{c}\right)=0$. Thus,

$$
\begin{aligned}
& \tau_{c}=\tau_{j_{c}}^{\left(n_{c}\right)}=\min \left\{\tau_{j}^{(n)}>0,0 \leq j \leq 2, n \geq 1\right\}, \\
& \omega_{c}=\omega_{j_{c}} .
\end{aligned}
$$

Theorem 3. For the time lag $\tau$, let the critical time lag $\tau_{c}$ and $\omega_{c}$ be defined as in (4.16), and suppose that $3 \omega_{c}^{6}+2 p \omega_{c}^{4}+q \omega_{c}^{2} \neq 0$ then the system of delay differential equations (4.1) exhibits the Hopf bifurcation at the steady state (3.5).

Proof. We will show that

$$
\left.\frac{d \alpha(\tau)}{d \tau}\right|_{\tau=\tau_{c}} \neq 0
$$

which guarantees that the Hopf bifurcation occurs. First, we equate real parts and imaginary parts of the right side of equation (4.8) to zero :

$$
\begin{gathered}
e^{-\tau \alpha}\left[\left(d_{2}+2 d_{1} \alpha\right) \omega \sin \omega \tau+\left[d_{3}+d_{2} \alpha+d_{1}\left(\alpha^{2}-\omega^{2}\right)\right] \cos \omega \tau\right]=\left(b_{1}+3 \alpha\right) \omega^{2}-b_{3}-b_{2} \alpha-b_{1} \alpha^{2}-\alpha^{3} \\
e^{-\tau \alpha}\left[\left(d_{1} \omega^{2}-d_{3}-d_{2} \alpha-d_{1} \alpha^{2}\right) \sin \omega \tau+\left(d_{2} \omega+2 d_{1} \alpha \omega\right) \cos \omega \tau\right]=\omega^{3}-b_{2} \omega-2 b_{1} \alpha \omega-3 \alpha^{2} \omega .
\end{gathered}
$$

We differentiate equations (4.18) and (4.19) with respect to $\tau$ and evaluate at $\tau=\tau_{c}$ for which $\alpha\left(\tau_{c}\right)=0$ and $\omega\left(\tau_{c}\right)=\omega_{c}$. We then obtain

$$
\begin{aligned}
& \left.E_{1} \frac{d \omega}{d \tau}\right|_{\tau=\tau_{c}}-\left.E_{2} \frac{d \alpha}{d \tau}\right|_{\tau=\tau_{c}}=E_{3} \cos \omega_{c} \tau_{c}+E_{4} \sin \omega_{c} \tau_{c} \\
& \left.E_{2} \frac{d \omega}{d \tau}\right|_{\tau=\tau_{c}}+\left.E_{1} \frac{d \alpha}{d \tau}\right|_{\tau=\tau_{c}}=E_{3} \sin \omega_{c} \tau_{c}-E_{4} \cos \omega_{c} \tau_{c}
\end{aligned}
$$

where

$$
\begin{gathered}
E_{1}:=2 b_{1} \omega_{c}+\left(2 d_{1} \omega_{c}-\tau_{c} d_{2} \omega_{c}\right) \cos \omega_{c} \tau_{c}+\left(\tau_{c} d_{3}-d_{2}-\tau_{c} d_{1} \omega_{c}^{2}\right) \sin \omega_{c} \tau_{c}, \\
E_{2}:=b_{2}-3 \omega_{c}^{2}+\left(d_{2}+\tau_{c} d_{1} \omega_{c}^{2}-\tau_{c} d_{3}\right) \cos \omega_{c} \tau_{c}+\left(2 d_{1} \omega_{c}-\tau_{c} d_{2} \omega_{c}\right) \sin \omega_{c} \tau_{c}, \\
E_{3}:=d_{2} \omega_{c}^{2} \text { and } E_{4}:=d_{1} \omega_{c}^{3}-d_{3} \omega_{c} .
\end{gathered}
$$

By solving equations (4.20) and (4.21) we have

$$
\left.\frac{d \alpha}{d \tau}\right|_{\tau=\tau_{c}}=\frac{\left(E_{1} E_{3}-E_{2} E_{4}\right) \sin \omega_{c} \tau_{c}-\left(E_{1} E_{4}+E_{2} E_{3}\right) \cos \omega_{c} \tau_{c}}{E_{1}^{2}+E_{2}^{2}} .
$$

From equations (4.10) and (4.11), we therefore obtain

$$
\begin{aligned}
\cos \omega_{c} \tau_{c} & =\frac{\left(b_{1} \omega_{c}^{2}-b_{3}\right)\left(d_{3}-d_{1} \omega_{c}^{2}\right)+d_{2} \omega_{c}\left(\omega_{c}^{3}-b_{2} \omega_{c}\right)}{d_{2}^{2} \omega_{c}^{2}+\left(d_{3}-d_{1} \omega_{c}^{2}\right)^{2}} \\
\sin \omega_{c} \tau_{c} & =\frac{d_{2} \omega_{c}\left(b_{1} \omega_{c}^{2}-b_{3}\right)-\left(d_{3}-d_{1} \omega_{c}^{2}\right)\left(\omega_{c}^{3}-b_{2} \omega_{c}\right)}{d_{2}^{2} \omega_{c}^{2}+\left(d_{3}-d_{1} \omega_{c}^{2}\right)^{2}} .
\end{aligned}
$$


Substituting equations (4.23) and (4.24) into equation (4.22) we then have

$$
\left.\frac{d \alpha}{d \tau}\right|_{\tau=\tau_{c}}=\frac{3 \omega_{c}^{6}+2 p \omega_{c}^{4}+q \omega_{c}^{2}}{E_{1}^{2}+E_{2}^{2}} \neq 0
$$

Hence, the Hopf bifurcation occurs when $\tau$ passes through the critical value $\tau_{c}$.

Corollary 1. Suppose $a_{1}, a_{2}, a_{5}>0$ and $a_{3}>1$. For $\tau_{c}$ and $\omega_{c}$ defined as in (4.16), if $p \geq 0$, $q \geq 0$, and either Lemma $2 i(a)$ or Lemma $2 i(b)$ is true, then the system of delay differential equations (4.1) exhibits the Hopf bifurcation at the steady state (3.5)

Proof. For $p \geq 0$ and $q \geq 0$ then $3 \omega_{c}^{6}+2 p \omega_{c}^{4}+q \omega_{c}^{2} \neq 0$. By Lemma 2 and Theorem 3, the Hopf bifurcation occurs.

\section{Numerical Results}

To show that the intracellular delay can effect the qualitative behavior of the three variables CD4+T-cell(x), HIV(y) and CTL(z), we conduct numerical simulations to confirm the theoretical predictions discussed in section 3 and section 4 . We first use the values of parameters $a_{1}$, $a_{2}, a_{3}, a_{4}$ and $a_{5}$ that are suggested by Verotta and Schaedeli [31] to give biologically realistic simulation results.

Example 1. To show the qualitative behavior of the three variables CD4+T-cell(x), HIV(y), and CTL(z) when the basic reproductive rate of the virus is under the control level and the intracellular delay is ignored, we numerically solve the system (3.2) by using the sixth order Runge-Kutta method with $a_{1}=0.133, a_{2}=0.85, a_{3}=0.278, a_{4}=4.56$, and $a_{5}=1.22$ (suggested in [31]).

In Figures 1a)-1c), we show the time series of the density of the CD4+T-cells in blood, the density of HIV in blood, and that of the CTL in blood, respectively. The CD4+T-cell density becomes constant as time passes, while the HIV density and CTL density both tend to zero.

Figure 1d) focuses on the behavior of the densities of HIV and CTL near the initial point to show the interaction between virus and immune response.

From the mathematical point of view, the numerical result illustrated in Figure 1 exhibits the same characteristics as those theoretically predicted in Proposition $2(i)$, that is when $a_{3}<1$ the trajectory tends to the steady state $(1,0,0)$. From the biological point of view, when $a_{3}<1$, the basic reproductive rate of the virus is under the control level, then at the beginning of the infection each virus cell produces on the average less than its decline rate. Hence, the infection cannot spread and finally the virus vanishes and the CD4+T-cell density becomes constant at $\delta_{1} / \delta_{3}$.

Example 2. To show the qualitative behavior of the three variables CD4+T-cell(x), HIV(y), and CTL(z) when the basic reproductive rate of the virus is under the control level and the intracellular delay is considered, we numerically solve the system (4.1) by using the sixth order Runge-Kutta method with $a_{1}=0.133, a_{2}=0.85, a_{3}=0.278, a_{4}=4.56, a_{5}=1.22$, and $\tau=0.1$.

Figures 2a)-2c) show the time series of CD4+T-cell density in blood, HIV density in blood, and CTL density in blood, respectively, for this case. The CD4+T-cell density becomes constant as time passes while HIV and CTL densities both tend to zero. 
Figure 2d) focuses on the behavior of the densities of HIV and CTL near the initial point to show the interaction between virus and immune response.

From the mathematical point of view, the numerical result illustrated in Figure 2 exhibits the same behavior as that theoretically predicted in Proposition 4(i), that is when $a_{3}<1$ the trajectory tends to the steady state $(1,0,0)$. Moreover, we would like to point out that under the same circumstance the delay effects the densities of the HIV and CTL in blood. As shown in Figure 1d) and Figure 2d) the maximum densities of HIV and CTL in Example 1 are higher and both HIV and CTL densities reach zero faster than those in Example 2. From the biological point of view, when $a_{3}<1$, the basic reproductive rate of the virus is under the control level, then at the beginning of the infection each virus cell produces on the average less than its decline rate. Hence, the infection cannot spread and finally the virus vanishes and CD4+T-cell density becomes constant at $\delta_{1} / \delta_{3}$. Due to the delay between the infection of a CD4+T-cell and the emission of viral particles on a cellular level, the virus density in blood is low hence the CTL density in blood is low too. However the infection cannot spread and finally the virus density goes to zero and CD4+T-cell density reaches constant value $\delta_{1} / \delta_{3}$.

Example 3. To show the qualitative behavior of the three variables CD4+T-cell(x), HIV(y), and CTL(z) when the basic reproductive rate of the virus is not under the control level and the intracellular delay is ignored, we numerically solve the system (3.2) by using the sixth order Runge-Kutta method with $a_{1}=0.0128, a_{2}=3.13, a_{3}=2.55, a_{4}=7.32$, and $a_{5}=4.08$.

In Figure 3c), we show the time series of the density of the CTL in blood, which oscillates before finally converging to a constant value.

Figure 3d) focuses on the behavior of the densities of HIV and CTL near the initial point to show the interaction between virus and immune response.

From the mathematical point of view, the numerical result illustrated in Figure 3 exhibits the same characteristics as those theoretically predicted in Proposition 2(ii), that is when $a_{3}>1$ the trajectory tends to the steady state $(0.73,0.36,0.36)$. From the biological point of view, when $a_{3}>1$, the basic reproductive rate of the virus is not under the control level, each virus cell produces on the average more than its loss rate. In this case, the virus can establish an infection.

Example 4. To show the qualitative behavior of the three variables CD4+T-cell(x), HIV(y), and $\operatorname{CTL}(\mathrm{z})$ when the basic reproductive rate of the virus is not under the control level and the intracellular delay is considered, we numerically solve the system (4.1) by using the sixth order Runge-Kutta method with $a_{1}=0.0128, a_{2}=3.13, a_{3}=2.55, a_{4}=7.32, a_{5}=4.08$, and $\tau=0.1$.

In Figures 4a)-4b), we show the time series of the density of the CD4+T-cell in blood, and that of the virus, respectively. Both are seen to become constant as time passes.

In Figure 4c), the time series of CTL density in blood shows oscillation before eventual convergence to the constant value.

Figure 4d) focuses on the behavior of the densities of HIV and CTL near the initial point to show the interaction between virus and immune response.

From the mathematical point of view, the numerical result illustrated in Figure 4 corresponds to the case where the conditions in Theorem 3 hold but $\tau<\tau_{c}$. Although $a_{3}>1$, the trajectory tends to the steady state $(0.73,0.36,0.36)$. Furthermore, we remark that under the same circumstance without delay, the densities of HIV and CTL in blood are effected. In Figure 3d), the oscillation 
frequency is lower and the amplitude is higher than those in Figure 4d). From the biological point of view, when $a_{3}>1$, the basic reproductive rate of the virus is not under the control level, each virus cell produces on the average more than its loss rate. In this case, the virus can establish an infection since the virus density changes very quickly, so that CTL is not able to get rid of the virus and therefore the infection persists, though it does not progress to full-blown AIDS.

Example 5. When the delay $\tau$ is large enough $\left(\tau>\tau_{c}\right)$, periodic behavior in all 3 state variables is predicted in Corollary 1. This is confirmed by the numerical solution shown in Figure 5.

\section{Discussion and Conclusion}

A mathematical non-linear differential equation model for HIV infection and immune response has been considered. The model has been extended by the inclusion of an intracellular delay effect. As mentioned by Herz et al. [14], this intracellular delay is of vital importance when the model is used to determine the half-life of free virus. Delays also affect the estimated value of the infected T-cell loss rate when it is assumed that the drug is not completely effective, as mentioned by Nelson et al. [19]. We believe that the delay effect leads to a model that is capable of more realistic physiology associated with a discrete time lag between the infection of a cell and the emission of viral particles. We refer the readers to [2] for biological data to compare with our model simulations.

Our goal is to improve the model and determine whether the stability of the steady state of the delay system (4.1) is effected by the introduction of a discrete time lag. We have analyzed the stability of the steady states of both model systems with and without delay, and conducted the numerical investigation to confirm the results. We have also derived the conditions for the existence of a Hopf bifurcation such that the steady state loses its stability at $\tau=\tau_{c}$. The question of stability is very important in all physical applications since we can never measure the initial conditions exactly. Therefore, we are interested in determining whether the steady states of the model system are stable or not. If the slightest disturbance completely alters the behavior of the system, then it is impossible to predict the long-term evolution of the system under consideration. With the parameter values used in the paper by Verotta and Schaedeli [31], our studies show that there does not exist such a critical delay beyond which the steady state would lose its stability resulting in oscillations.

In the biological sense Proposition 4(i) says that the delay will not effect the stability of the steady state (3.4) as long as the basic reproductive ratio for the virus is under the control level, $a_{3}<1$. Hence, this will result in virus clearance. CTL kills infected cells before they can produce sufficient amounts of viral progeny which finally results in the absence of the virus.

If the conditions in Theorem 1 are satisfied the infected steady state (3.5) of delay system (4.1) remains stable and we can conclude that incorporating a discrete intracellular time delay into the system (3.2) does not change the stability of the infected steady state (3.5). In other words, even if the immune response is maintained, virus persists. However the virus may persist without causing disease if the immune system is strongly responsive. This case keeps CD4+T-cell, viral load, and the immune responses low.

However, if Theorem 3 holds then the stability of the infected steady state (3.5) of delay model 
(4.1) depends on the delay value and the delay can induce oscillation in the number of CD4+ Tcells, HIV, and CTL. If the number of CD4+ T-cells is lower than 200 per $\mu l$, the virus may escape the control of the CTL and then full blown AIDS results but if the CD4+ T-cell wins out then the virus population is annihilated.

We would like to mention that these models focus on HIV infection and immune response which is in reality more complicated due to the fact that HIV mutates into many different forms over time. However, our model is able to provide a predictive value of the critical delay $\tau_{c}$ beyond which the system stability is lost, something that a more complicated, and more mathematically untractable, model will not allow. The existence of $\tau_{c}$ essentially implies that if the virus does not take too long (low delay $\tau$ ) between cell infection and emission of viral particles, then the viral proliferation can be kept at a manageable level. This might sound unreasonable on the surface, but we can attribute this effect to the role of the CTL which has been incorporated into our model. If, say, the amount of virus is perturbed to be above the steady state level, the rate of production of CTL will increase and killing rate of virus by CTL will subsequently increase. This results in a drop in the virus load. If the virus takes a long time to mature in the host cells $\left(\tau>\tau_{c}\right)$, the viral depletion may continue till a low level is reached. CTL's action is then correspondingly low, allowing time for the virus to regenerate. In the case that $a_{3}>1$, the high basic reproduction ratio means that the viral load can increase very quickly. If the emission of new viral particles from host cells now occurs at the appropriate time, then virus load can increase very quickly and overshoots the previous high level. This leads to oscillation in the density of infective virus, and for high enough $a_{3}$ and appropriately high delay $\tau$, the quick surges of viral load in an unbounded fashion can eventuate, characteristic of full-blown AIDS.

On the other hand, if the emission delay can be kept small, the action of CTL is responding to the level of virus density that reflects more or less the current situation, with very small inherent delay, then our immune response mechanism will be able to keep the infection at a steady level, perhaps as a long-term nonprogressor, eventhough it cannot be completely eradicated, if $\bar{y}$ is not too high and $\bar{x}$ is not too low.

Figure 6 a) shows the time series of the CD4 count and viral load in the case where the reproduction ratio $a_{3}$ is not too high and $a_{4}$ is not too low. The viral load peaks in the first few days then drops and bottoms out, reaching and remaining at the relatively low steady state value for all subsequent time. The CD4 count in this case remains sufficiently high. This is then characteristic of a long-term non-progressor.

Figure $6 \mathrm{~b}$ ), however, shows the case in which $a_{3}$ is high and $a_{4}$ is very low indicative of an inefficient immune system. The number of CD4 initially drops sharply then slowly increases, maintaining a level that is not too low during the dormant period. The viral load, after the initial surge and a dormant period, starts to increase while the CD4 count sharply decreases, dropping to a level which is too low (less than about 200 per $\mathrm{ml}$ ). This is then the case in which progression to full blown AIDS can be expected, and the patient can succumb to opportunistic infection.

The above analytical interpretation has important implications in our attempts at therapy as well as drug development. If the emissions of viral particles from the host cells could be tuned and the killer cells CTL can somehow be effectively controlled, then it has been suggested by our model analysis that a patient's immunological system could be assisted in winning over the battle. 
Such valuable insights can be gained from theoretical analysis of a model that has been kept from being too complicated. In so doing, we have traded off the accuracy that might be gained from a larger model that considers more variable factors and compartments, and this will be a subject for our future endeavor.

\section{Acknowledgements}

The research of this paper was performed at Syracuse University and was supported by the National Research Council of Thailand, the National Center for Genetic Engineering and Biotechnology, and the Faculty of Science, Chiangmai University. It was supported in part by the US National Science Foundation under grant CCR-0312113, and by the Natural Science Foundation of China under grant 10371122. 

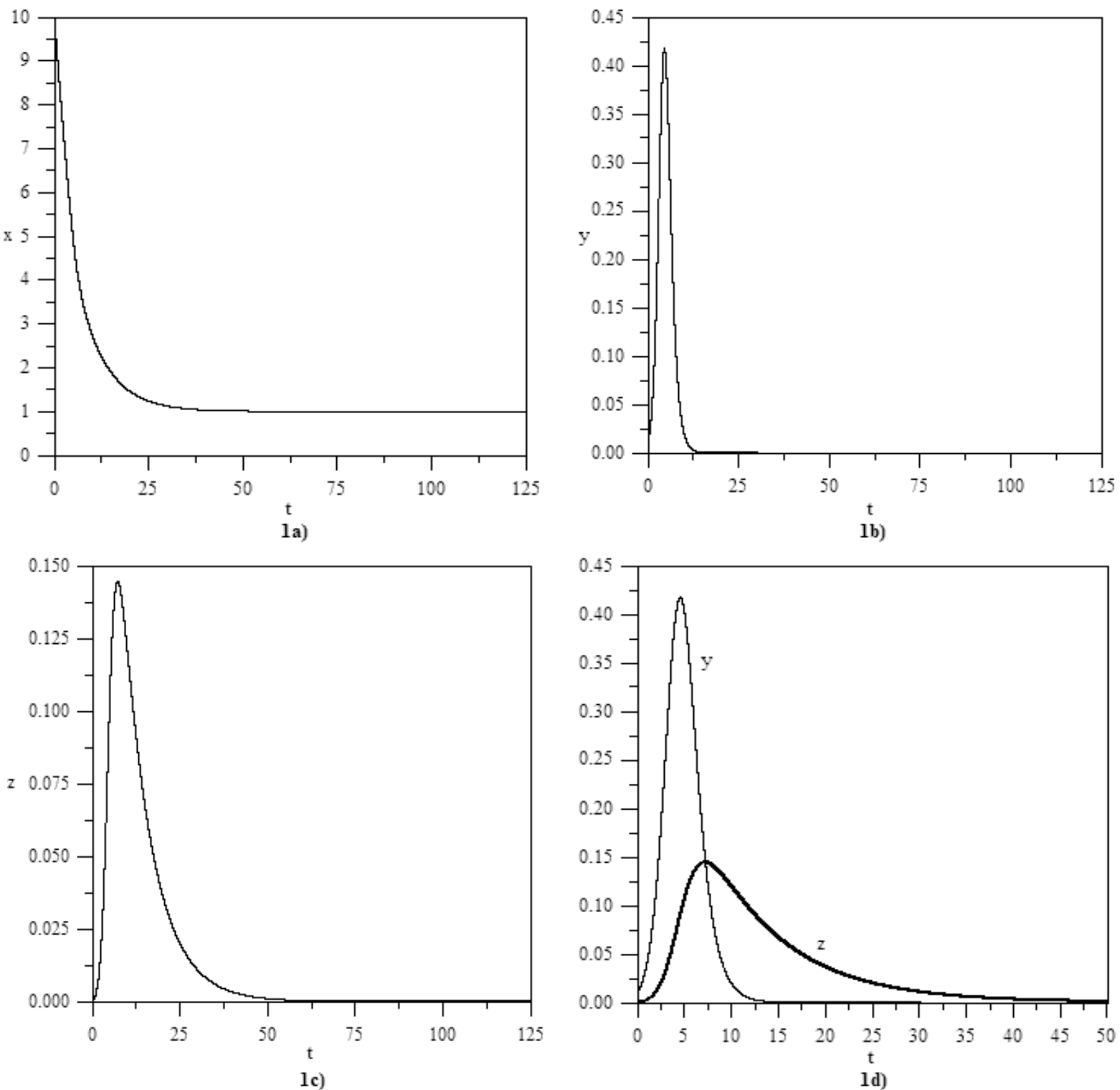

Figure 1: Numerical simulation of the model system (3.2) where parameter values, $a_{1}=0.133, a_{2}=0.85$, $a_{3}=0.278, a_{4}=4.56$, and $a_{5}=1.22$, satisfy conditions in Proposition 2(i). Figure 1a) shows the time series of the density of CD4+T-cells which tends to a constant value, while 1b) and 1c) show that of HIV, and that of CTL in blood, respectively, which tend to zero as time passes. Figure 1d) shows the time series of HIV and CTL densities near the initial point. 

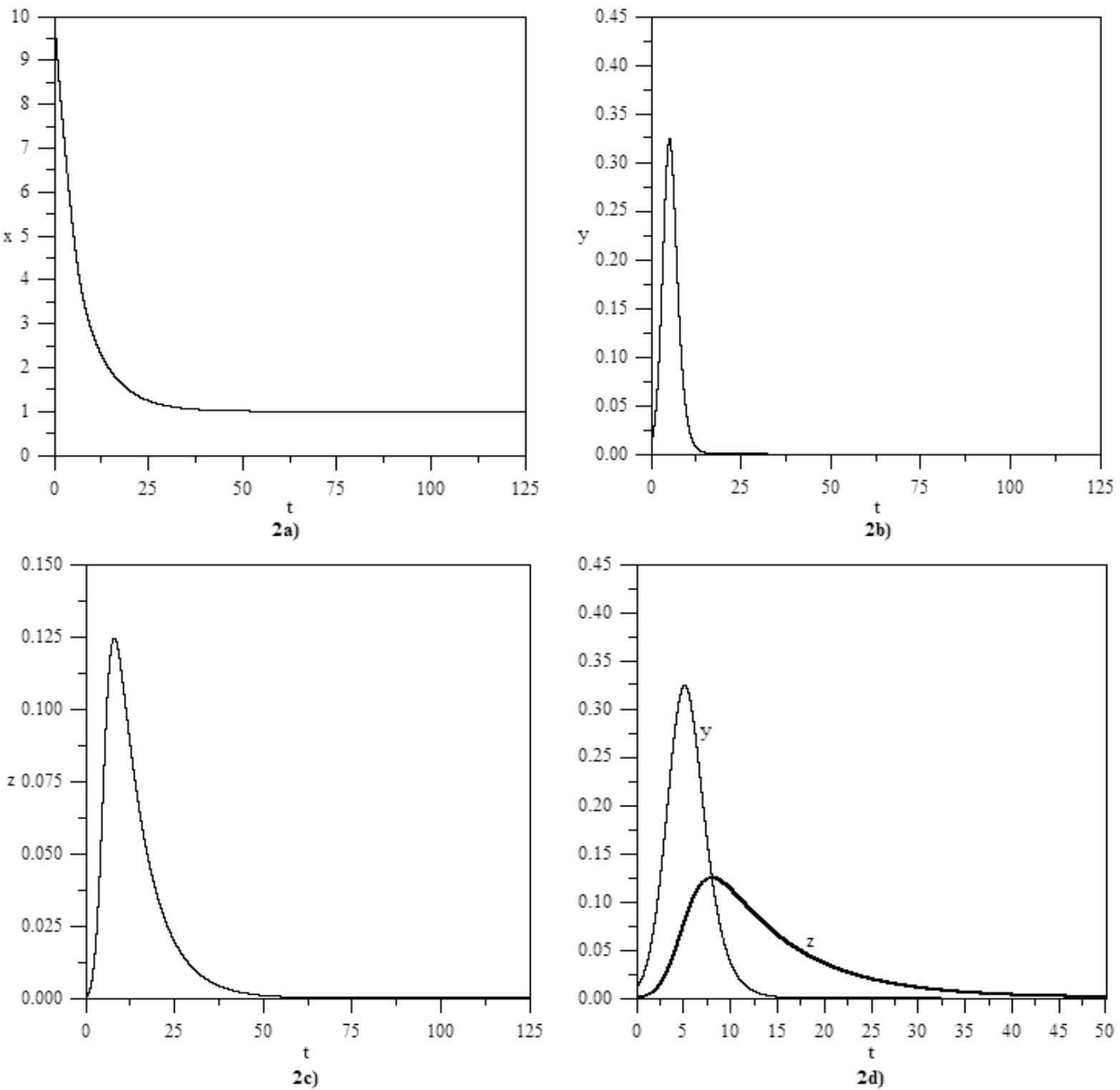

Figure 2: The numerical simulation of the model system (3.2) where $a_{1}=0.0128, a_{2}=3.13, a_{3}=2.55$, $a_{4}=7.32$, and $a_{5}=4.08$, which correspond to the case (ii) in Proposition 2. Figure 2a) shows the time series of the density of CD4+T-cells which tends to a constant value while $2 \mathrm{~b}$ ) and $2 \mathrm{c}$ ) show that of HIV, and that of CTL in blood, respectively, which tend to zero as time passes. Figure 2d) shows the time series of HIV and CTL densities near the initial point. 

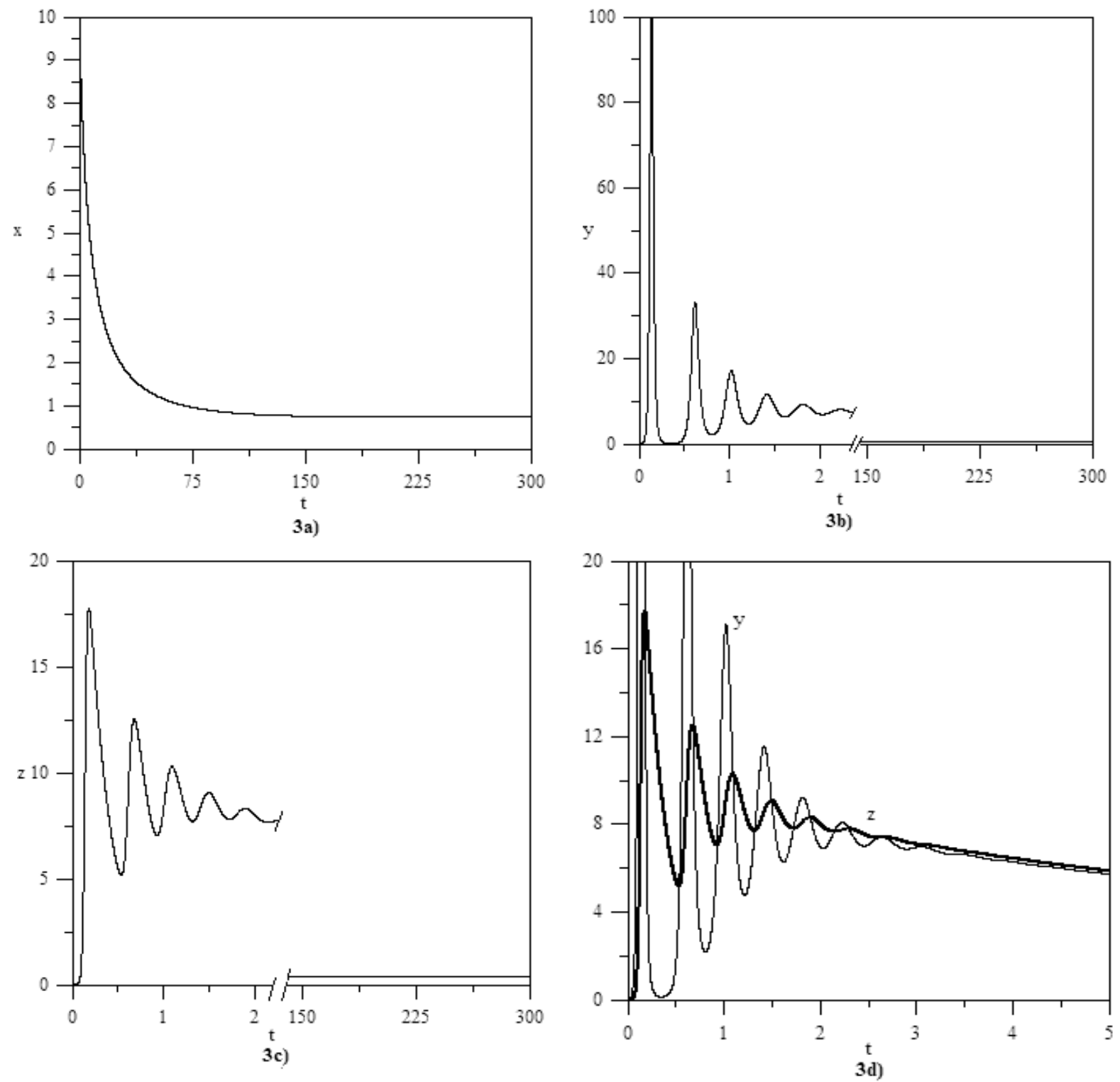

Figure 3: The numerical simulation of the model system (3.2) where $a_{1}=0.0128, a_{2}=3.13, a_{3}=2.55$, $a_{4}=7.32$, and $a_{5}=4.08$, which correspond to the case (ii) in Proposition 2. In 3a) the density of CD4+T cells tends to a constant value, in 3b) and 3c) that of HIV and CTL oscillates before converging to constant values. In 3d) HIV and CTL densities are shown near the initial point. 

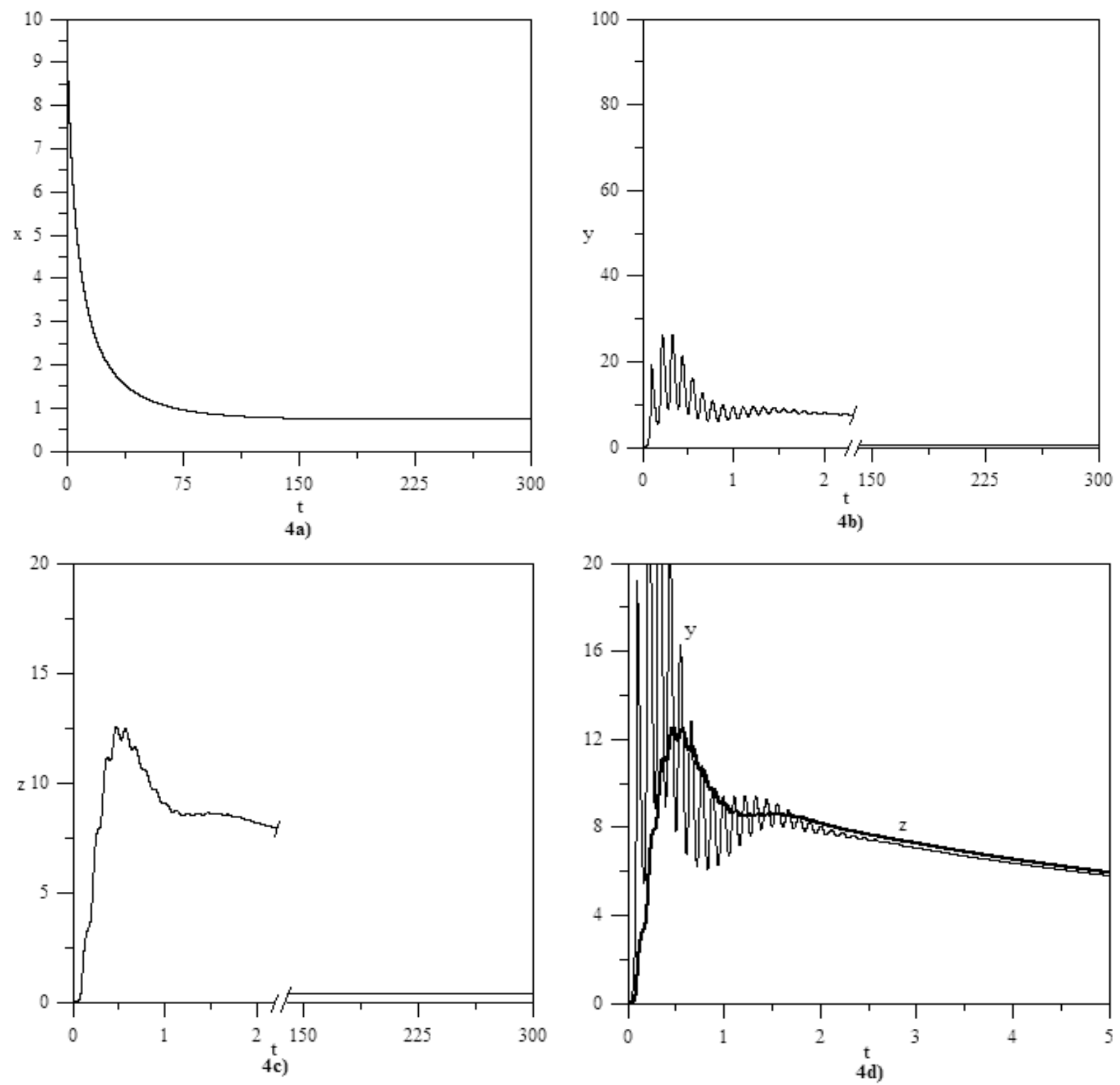

Figure 4: Numerical simulation of the delay model system (4.1) where parameter values, $a_{1}=0.0128$, $a_{2}=3.13, a_{3}=2.55, a_{4}=7.32, a_{5}=4.08$, and $\tau=0.1$, satisfy the requirement in Theorem 1. In $\left.4 \mathrm{a}\right)$ the density of CD4+T cells tends to a constant value, in $4 \mathrm{~b}$ ) and $4 \mathrm{c}$ ) those of HIV and CTL oscillate before converging to constant values. In 4d) HIV and CTL densities are shown near the initial point. 


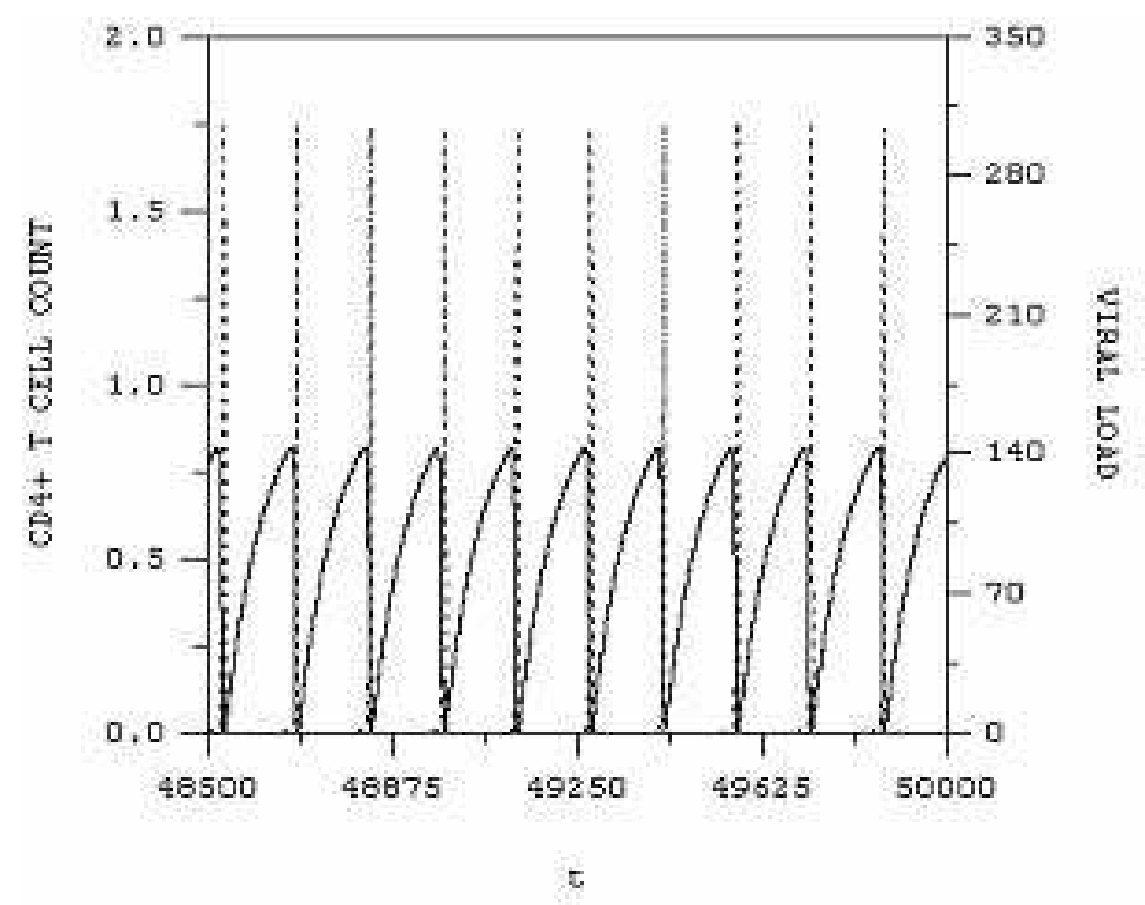

Figure 5.

Figure 5: Numerical simulation of the model system (3.2) showing sustained oscillation in the CD4+T-cell count and viral load as theoretically predicted in Corollary 1. Here, $a_{1}=0.0128, a_{2}=7.0, a_{3}=2.55$, $a_{4}=0.0001, a_{5}=4.08$, and $\tau=5$. 

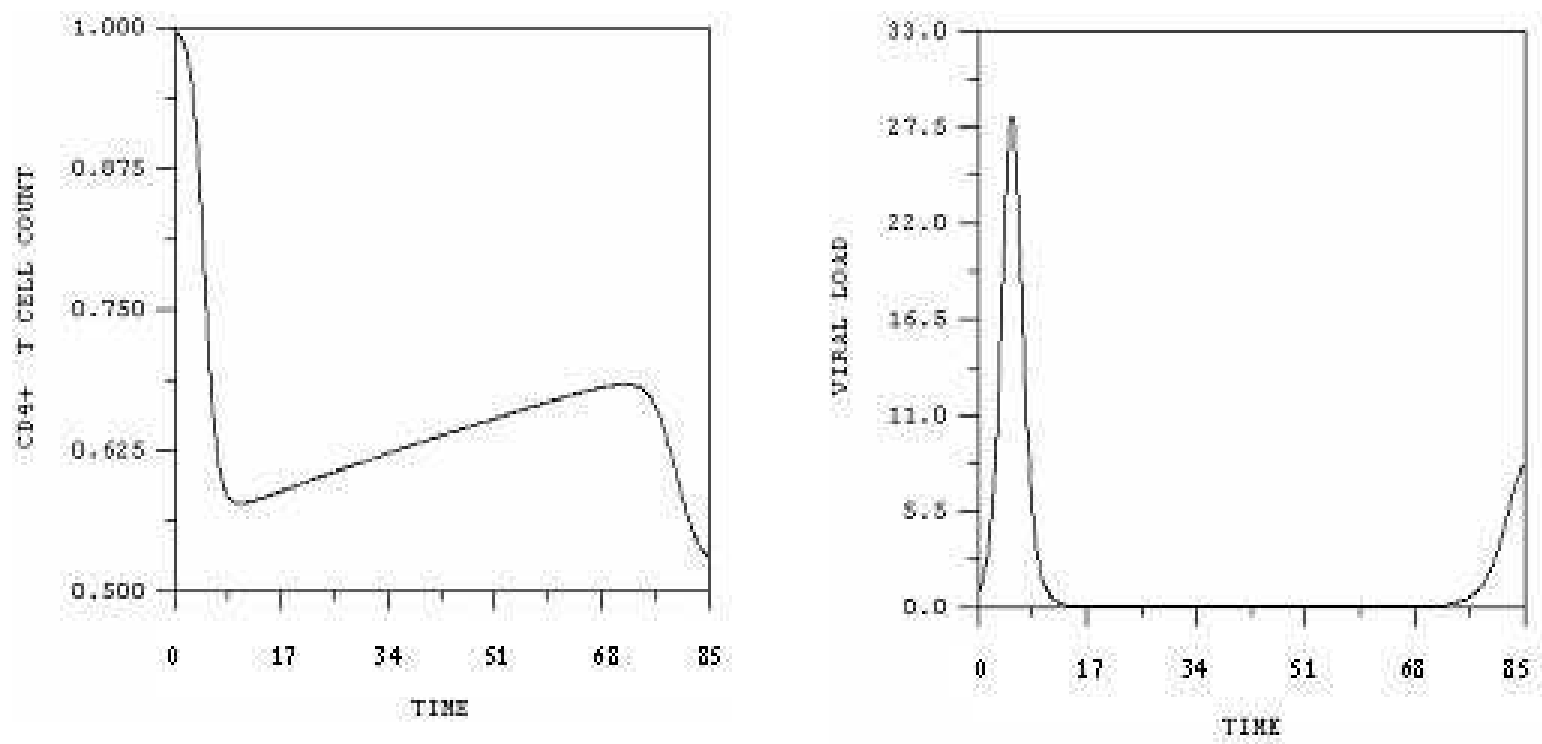

6a)
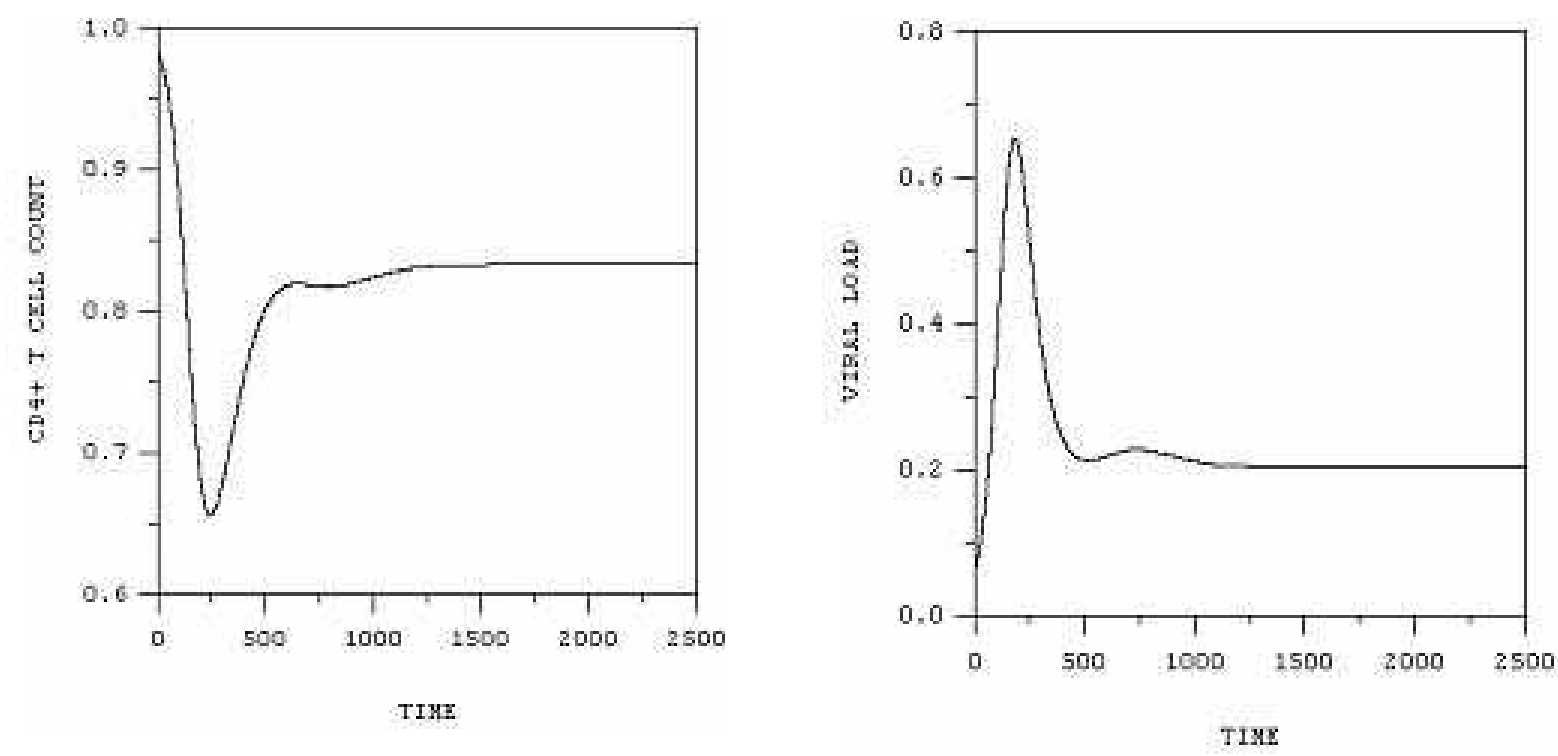

6h)

\section{Figure 6.}

Figure 6: Time series of $x$ (CD4+T-cell count), and $y$ (viral load) characteristic of a) progression to full blown AIDS, $a_{1}=0.0128, a_{2}=3.13, a_{3}=2.55, a_{4}=0.362, a_{5}=4.08, \tau=0.1$, and $\mathrm{b}$ ) a long-term nonprogressor, $a_{1}=0.005, a_{2}=0.2, a_{3}=7.0, a_{4}=0.5, a_{5}=0.03, \tau=0.1$. In 6 a) the C44+T cell count drops to very low level while the viral load inogases after the latent period to develop to full blown AIDS. In $6 \mathrm{~b}$ ) the CD4+T cell count retain a constant level while the viral load remains low in a long-term non-progressor. 


\section{References}

[1] R.A. Arnaout, M.A. Nowak, M. A. Competitive coexistence in antiviral immunity. J. Theor. Biol., 204 (2000), 431-441.

[2] S. Bajaria, G. Webb, D. Kirschner. Predicting differential responses to structured treatment interruptions during HAART. Bulletin of Mathematical Biology, 66 (2000), 1093-1118.

[3] R. Bellman, K.L. Cooke. Differential-difference equations. New York: Academic Press Inc, 1963.

[4] S. Bonhoeffer, J.M. Coffin, M.A. Nowak. Human immunodeficiency virus drug therapy and virus load. J. Virol., 71 (1997) 3275-3278.

[5] J. Chen, M. Cloyd. The potential importance of HIV-induction of lymphocyte homing to lymph nodes. Int. Immunol., 11 (1999), 1591-1594.

[6] R.I. Connor, H. Mohri, Y. Cao, D.D. Ho. Increased viral burden and cytopathicity correlate temporally with CD4+ T-lymphocyte decline and clinical progression in human immunodeficiency virus type 1-infected individuals. J. Virol., 67 (1993), 1772-1777.

[7] R.V. Culshaw, S. Ruan. A delay-differential equation model of HIV infection of CD4+ T-cells. Math. Biosci., 165 (2000), 27-39.

[8] R.D. Driver. Ordinary and delay differential equations. Springer-Verlag, New York, 1932.

[9] L. Edelstein-Keshet. Mathematical Model in Biology. Random House, New York, 1998.

[10] N.M. Ferguson, F. de Wolf, A.C. Ghani, C. Fraser, C.A. Donnelly, P. Reiss, J.M.A. Lange, S.A. Danner, G.P. Garnett, J. Goudsmit, R.M. Anderson. Antigen-driven CD4+ T cell and HIV-1 dynamics: residual viral replication under highly active antiretroviral therapy. PNAS, 96 (1999), 15167-15172.

[11] Z. Grossman, M. Polis, M.B. Feinberg, I. Levi, S. Jankelevich, R. Yarchoan, J. Boon, F. de Wolf, J.M. Lange, J. Goudsmit, D.S. Dimitrov, W.E. Paul. Ongoing HIV dissemination during HAART. Nat. Med., 5 (1999), 1099-1104.

[12] A. Halanay. Differential equations: stability, oscillation, time lags. Academic Press Inc, New York, 1966.

[13] B.D. Hassard, N.D. Kazarinoff, Y.H. Wan. Theory and applications of Hopf bifurcation. Cambridge University Press, New York, 1981.

[14] A.V.M. Herz, S. Bonhoeffer, R.M. Anderson, R. M. May, M.A. Nowak. Viral dynamics in vivo: limitations on estimates of intracellular delay and virus decay. Proc. Nat. Acad. Sci. USA, 93 (1966), 7247-7251. 
[15] Y. Lenbury, R. Ouncharoen, N. Tumrasvin. Higher-dimensional separation principle for the analysis of relaxation oscillations in nonlinear systems: application to a model of HIV infection. IMA J. Math. Appl. Med. Biol., 17 (2000), 243-261.

[16] N. MacDonald. Time lags in biological model. Springer, Berlin, 1970.

[17] J.E. Marsaen, M. McCracken. The Hopf bifurcation and its applications. Springer-Verlag, New York, 1976.

[18] J.E. Mittler, B. Sulzer, A.U. Neumann, A.S. Perelson. Influence of delayed viral production of viral dynamics in HIV-1 infected patients. Math. Biosci., 152 (1998), 143-163.

[19] P.W. Nelson, J.D. Murray, A.S. Perelson. A model of HIV-1 pathogenesis that includes an intracellular delay. Math. Biosci., 163 (2000), 201-215.

[20] P.W. Nelson,A.S. Perelson. Mathematical analysis of delay differential equation models of HIV-1 infection. Math. Biosci., 179 (2002), 73-94.

[21] P. Nelson, J. Mittler, A. Perelson. Effect of drug efficacy and the eclipse phase of the viral life cycle on estimates of HIV-1 viral dynamic parameters. J. AIDS., 26 (2001), 405-412.

[22] M.A. Nowak, C.R.M. Bangham. Population dynamics of immune responses to persistent viruses. Science, 272 (1996), 74-79.

[23] A.S. Perelson, D.E. Kirschner, R. De Boer. Dynamics of HIV infection of CD4+ T-cells. Math. Biosci., 114 (1993), 81-125.

[24] A.S. Perelson. Modelling the interaction of HIV with the immune system. In: Mathematical and Statistical Approaches to AIDS Epidemiology. C. Castillo-Chavez, ed., Springer, New York, 1989.

[25] A.S. Perelson. Modelling viral and immune system dynamics. Nature Reviews Immunology, 2 (2002), 28-36.

[26] A.S. Perelson, A.U. Neumann, M. Markowitz. HIV-1 dynamics in vivo: virion clearance rate, infected cell life-span, and viral generation time. Science, 271 (1996), 1582-1586.

[27] M. Piatak Jr., M.S. Saag, L.C. Yang, S.J. Clark, J.C. Kappes, K.C. Luk, B.H. Hahn, G.M. Shaw, J.D. Lifson. High levels of HIV-1 in plasma during all stages of infection determined by competitive PCR. Science, 259 (1993), 1749-1754.

[28] O. Plaat. Ordinary differential equations. Holden Day, San Francisco, 1971.

[29] S. Ruan, J. Wei. On the zeros of a third degree exponential polynomial with applications to a delayed model for the control of testosterone secretion. IMA J. Math. Appl. Med. Biol., 18 (2001), No. 1, 41-52. 
[30] J. Tam. Delay effect in a model for virus replication. IMA J. Math. Appl. Med. Biol., 16 (1999), 29-37.

[31] D. Verotta, F. Schaedeli. Non-linear dynamics models characterizing long-term virological data from AIDS clinical trials. Math. Biosci., 176 (2002), 163-183.

[32] R.M. Zinkernagel. Immunology taught by viruses. J. Science, 271 (1996), 173-178. 